\title{
Effects of quantized fields on the spacetime geometries of static spherically symmetric black holes
}

\author{
Paul R. Anderson \\ Department of Physics, Wake Forest University, \\ P.O. Box 7507, Winston-Salem, NC, 27109, U.S.A. and \\ Racah Institute of Physics, Hebrew University of Jerusalem, \\ Givat Ram, Jerusalem, 91904, Israel and \\ Departamento de Física Teórica and IFIC, Universidad de Valencia-CSIC, \\ C. Dr. Moliner 50, Burjassot-46100, Valencia, Spain \\ Mathew Binkley \\ Department of Physics, Wake Forest University, \\ P.O. Box 7507, Winston-Salem, NC, 27109, U.S.A. \\ Hector Calderon and William A. Hiscock \\ Department of Physics, Montana State University, Bozeman, MT, 59717, U.S.A. \\ Emil Mottola \\ Theoretical Division, T8, Los Alamos National Laboratory, Los Alamos, NM, 87545, U.S.A. \\ Ruslan Vaulin \\ Department of Physics, Florida Atlantic University, \\ 777 Glades Road, Boca Raton, FL, 33431, U.S.A.

\begin{abstract}
Analytic approximations for the stress-energy of quantized fields in the Hartle-Hawking state 1] in static black hole spacetimes predict divergences on the event horizon of the black hole for a number of important cases. Such divergences, if real, could substantially alter the spacetime geometry near the event horizon, possibly preventing the black hole from existing. The results of three investigations of these types of effects are presented. The first involves a new analytic approximation for conformally invariant fields in Reissner-Nordström (RN) spacetimes which is finite on the horizon. The second focuses on the stress-energy of massless scalar fields in Schwarzschild-de Sitter black holes. The third focuses on the stress-energy of massless scalar fields in zero temperature black hole geometries that could be solutions to the semiclassical backreaction equations near the event horizon of the black hole.
\end{abstract}

PACS numbers: 
It is well known that previous analytical approximations for quantized massless fields in the Hartle-Hawking state in RN spacetimes [2, 3] predict that one component of the stress-energy tensor diverges logarithmically on the event horizon in the nonextreme case. For extreme ReissnerNordström (ERN) black holes there is both a powerlaw and a logarithmic divergence predicted. On the other hand numerical calculations for massless spin 0 and spin $1 / 2$ fields [3, 4, [5] show no evidence for such divergences.

For conformally invariant fields there is an approximate effective action, sometimes called the anomaly action, which when varied with respect to the metric results in a stress-energy tensor whose trace is equal to the trace anomaly [6, 7, 8, 9]. The behavior of this stress-energy tensor has been studied for Schwarzschild and other spacetimes [10, 11]. It can be written in terms of two auxiliary fields which both obey fourth order differential equations. This results in numerous solutions which, at least to some extent, correspond to various possible states for the quantized fields [10, 11].

An investigation of this action for the case of RN and ERN spacetimes [12] shows that there exist solutions to the auxiliary field equations which give a finite stress-energy on the event horizons of both types of black holes. The best approximation can be obtained by fitting the value of $T^{t}{ }_{t}$ on the horizon and at large $r$ in RN. This results in one free parameter which can be varied to give the best fit to the stress-energy at intermediate locations. The approximation for ERN is not as good because $T^{t}{ }_{t}$ is fixed on horizon and the value is not close to the correct one. However, it is still possible to fix the value of $\left(T_{r}^{r}-T^{t} t\right) /\left(-g_{t t}\right)$ on the horizon and to fix $T^{t}{ }_{t}$ at large $r$. While not perfect, these approximations have the advantage of being the only known approximations for massless quantized fields which do not have divergences on the horizon for RN and ERN.

The analytic approximation [3] for massless scalar fields with arbitrary coupling $\xi$ to the scalar curvature $R$ makes the somewhat surprising prediction [13] that if the black hole temperature is nonzero and if $R \neq 0$ on the horizon (e.g. in Schwarzschild-de Sitter spacetimes), then near the horizon

$$
\left\langle T^{t}{ }_{t}\right\rangle \sim-\left\langle T_{r}^{r}\right\rangle \sim \frac{\xi(\xi-1 / 6)}{r_{h}\left(r-r_{h}\right)} R\left(r_{h}\right)
$$

These components are finite on the horizon in both RN and ERN spacetimes where $R=0$. Given the fact that divergences predicted for a different component of the stress-energy tensor in $\mathrm{RN}$ and ERN turned out not to be real, one might expect that the same would be true for these apparent divergences associated with $R \neq 0$.

To test this conjecture, numerical computations of the stress-energy tensor for massless scalar 
fields with various values of $\xi$ have been computed on the horizon for Schwarzschild-de Sitter spacetimes [13]. In these spacetimes there is both an event horizon for the black hole and a cosmological horizon and the two are at different temperatures. However, the fields can be put in the Hartle-Hawking state if the black hole is surrounded by a perfectly reflecting mirror. The numerical calculations have been done using a variation of a method [14] developed for Schwarzschild spacetime that gives the values of $\left\langle T^{t}{ }_{t}\right\rangle,\left\langle T_{r}^{r}\right\rangle$, and $\left\langle T^{\theta}{ }_{\theta}\right\rangle$ on the horizon. The numerical evidence indicates that these components are finite on the event horizon.

The existence of the trace anomaly, which is nonzero for Schwarzschild and RN spacetimes, means that $R \neq 0$ for self-consistent solutions to the semiclassical backreaction equations. The case of zero temperature black holes is a particularly natural one to investigate because there is no mirror needed to contain the radiation as there is for other black holes when the fields are in the Hartle-Hawking state.

The existence of a divergence in the stress-energy of a potential solution to the semiclassical equations would likely prevent that solution from existing. However, that does not necessarily mean that no zero temperature black hole could exist. Recent results regarding zero temperature black holes in two dimensions [15, 16] show that it is possible that the divergences could imply that no static state exists but that there is no divergence if the black hole forms from gravitational collapse with the fields in a particular nonstatic state. Nevertheless, this is still a significant deviation from one's intuition that static solutions to the semiclassical backreaction equations should exist that describe zero temperature black holes.

To determine the effects of the fields on the spacetime geometry in as complete a way as possible, it is useful to work in the context of a large $N$ expansion where $N$ is the number of identical quantized scalar fields. Unlike the usual loop expansion, the leading order terms in the large $N$ expansion allow for the possibility that quantum effects can significantly alter the spacetime geometry. It is also consistent to neglect the graviton stress tensor since this comes in at next to leading order.

If only conformally invariant fields are present then it is possible to argue that static spherically symmetric zero temperature black hole metrics near the event horizon should have the general form

$$
d s^{2}=-\left[a_{2}\left(r-r_{h}\right)^{2}+\ldots\right] d t^{2}+\left[b_{2}(r-r h)^{2}+\ldots\right]^{-1}+r^{2} d \Omega^{2}
$$

with $r_{h}$ the radius of the event horizon and $a_{2}$ and $b_{2}$ positive constants. The trace equation has been solved in this case and it has been found that $b_{2}$ is a function of $r_{h}$ and that $b_{2}>1$ [17]. There is good evidence that $\left\langle T^{\mu}{ }_{\nu}\right\rangle$ depends only on geometry near the horizon. Numerical results 
have been obtained for the exact metric $-g_{t t}=\left(r-r_{h}\right)^{2} / r^{2}$ and $g^{r r}=b_{2} g_{t t}$ for various values of $b_{2}$.

In all cases considered so far, there is preliminary evidence that the stress-energy of the conformally invariant scalar field diverges on the horizon. The divergence appears to occur for all values of $b_{2}>1$. However this is not conclusive since the value of the component which diverges on the horizon depends on $a_{3}, b_{3}$ and possibly higher terms in the expansion. In the cases where the divergence is present it occurs for all $r_{0}>0$ and thus for macroscopic as well as microscopic black holes.

In summary, a new analytic approximation has been found for Reissner-Nordström and extreme Reissner-Nordström spacetimes which is finite on the event horizon. No evidence has been found for the existence of divergences predicted by an analytical approximation for the stress-energy of quantized massless scalar fields in nonzero temperature black hole spacetimes when $R \neq 0$. There is evidence that spacetime geometries exist for which the stress-energy of the conformally invariant scalar field diverges on the event horizon of a zero temperature black hole. However the possibility that there are self-consistent static zero temperature black hole solutions to the semiclassical backreaction equations has not been ruled out.

\section{Acknowledgments}

P.R.A. would like to thank Jacob Bekenstein, Alessandro Fabbri, Sara Farese, and José NavarroSalas for helpful conversations. This research has been partially supported by grant numbers PHY-9800971, PHY-0070981, and PHY-0556292 from the National Science Foundation. P.R.A. thanks the Gravitation Group at the University of Maryland for hospitality and acknowledges the Einstein Center at Hebrew University, the Forchheimer Foundation, and the Spanish Ministerio de Educación y Ciencia for financial support.

[1] J. B. Hartle and S. W. Hawking, Phys. Rev. D 13, 2188 (1976).

[2] V. P. Frolov and A. I. Zel'nikov, Phys. Rev. D 35, 3031 (1987).

[3] P.R. Anderson, W.A. Hiscock and D.A. Samuel, Phys. Rev. Lett. 70, 1739 (1993); Phys. Rev. D 51, 4337 (1995).

[4] P. R. Anderson, W. A. Hiscock, and D. J. Loranz, Phys. Rev. Lett. 74, 4365 (1995).

[5] E. D. Carlson, W. H. Hirsch, B. Obermayer, P. R. Anderson, and P. B. Groves, Phys. Rev. Lett. 91, 051301 (2003). 
[6] R. J. Riegert. Phys. Lett. 134B, 56 (1984).

[7] E. S. Fradkin and A. A. Tseytlin. Phys. Lett. 134B, 187 (1984).

[8] S. D. Odintsov and I. L. Shapiro. Class. Quant. Grav. 8, L57 (1991).

[9] P. O. Mazur and E. Mottola. Phys. Rev. D 64, 104022 (2001).

[10] R. Balbinot, A. Fabbri, and I. Shapiro. Phys. Rev. Lett. 83, 1494 (1999); Nucl. Phys. B559 301 (1999).

[11] E. Mottola and R. Vaulin, Phys. Rev. D 74, 064004 (2006).

[12] P. R. Anderson, E. Mottola, and R. Vaulin, manuscript in preparation.

[13] H. Calderon, W. A. Hiscock, and P. R. Anderson, manuscript in preparation.

[14] P. Candelas, Phys. Rev. D 21, 2185 (1980).

[15] R. Balbinot, S. Fagnocchi, A. Fabbri, S. Farese, J. Navarro-Salas, Phys. Rev. D 70, 064031 (2004).

[16] S. Fagnocchi and S. Farese, Phys. Rev. D72, 024015 (2005).

[17] P. R. Anderson and C. D. Mull, Phys. Rev. D59, 044007 (1999); gr-qc/9711063 gr-qc/9707026. 